\title{
Synthesis and antimicrobial evaluation of 4-aryl-4,5-dihydro- 1H-1,5-benzodiazepin-2-ol derivatives
}

\section{Estael Ochoa ${ }^{2}$, Claudia Canaan ${ }^{2}$, Yamila Verdecia ${ }^{2}$, Edna Dória Peralta ${ }^{1}$, Osmar Calderón ${ }^{2}$, Raúl Ramos ${ }^{2}$, Yeily Seruto ${ }^{3}$, Aristóteles Góes Neto ${ }^{4}$, Heiddy Marquez Alvarez $^{1}$, Angélica Maria Lucchese ${ }^{1^{*}}$}

\author{
1. Laboratório de Química de Produtos Naturais e Bioativos. Universidade Estadual de Feira de Santana, \\ Brasil. \\ 2. Laboratorio de Síntesis Orgánica. Facultad de Química, Universidad de La Habana, Cuba. \\ 3. Instituto Cubano de Investigaciones de Derivados de La Caña de Azucar - ICIDCA, Cuba. \\ 4. Laboratório de Pesquisa em Microbiologia. Universidade Estadual de Feira de Santana, Brasil.
}

\section{*angelica.lucchese@gmail.com}

Keywords:1,5-benzodiazepines; microwave; antimicrobial.

\section{INTRODUCTION}

Benzodiazepines (BDZ) derivatives constituted the first pharmacological entity coined with the term privileged structure, due to their large number of therapeutic and pharmacological properties ${ }^{1,2}$. Besides the well-known Nervous Central System related properties (ansiolytic, hypnotic and anticonvulsant, among others), they had been found also useful as antibiotic, antiviral (HIV-1), antitumor or cardiovascular drugs ${ }^{1}$. In the other hand 1,5benzodiazepine moiety had been much less studied than 1,4-benzodiazepines counterparts.

In this work, it is reported the synthesis, characterization and antimicrobiological evaluation of different 4-aryl-4,5-dihydro-1H-1,5-benzodiazepin2-ol derivatives.

\section{RESULTS AND DISCUSSION}

The synthesis was carried out starting from the corresponding aryldenmalonate of Meldrum's acid and two different o-phenylendiamine derivatives, using ethanol as solvent, under reflux conditions. Final products with moderate yields in approximately $6 \mathrm{~h}$ of reaction are obtained. In order to account for an eco-friendly approach, the synthesis was carried out using water as solvent, as well as microwave activation energy (see Figure 1).

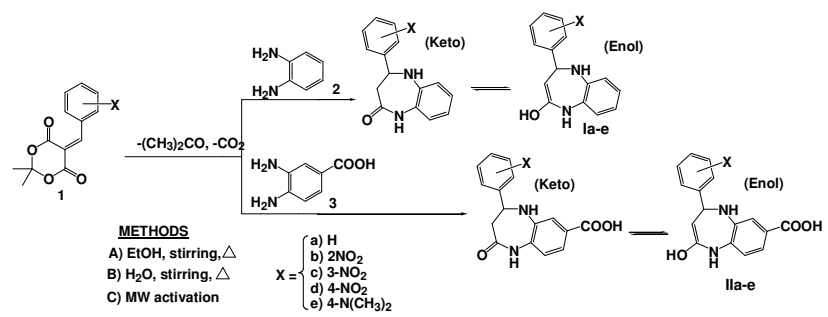

Figure 1. Synthetic scheme.
All products were characterized by mean of NMR experiments, IR, UV spectroscopy and mass spectrometry. The corresponding melting points are also reported. By mean of NMR experiments, it was possible to determine that enol (see Figure 1) is the prevailing tautomer form in solution. DFT quantum mechanics calculations also support this finding.

Selected derivatives were submitted into antimicrobial testing for bacteria (Escherichia coli CCMB261, Staphylococcus aureus, CCBM 262, Pseudomonas aeruginosa CCMB 268, Salmonella choleraesuis CCMB 281, Bacillus cereus CCMB 282, Micrococcus luteus CCMB 283) according to CLSI 2003 regulations, using resazurine as developer. Blue color indicates inhibition while pink color indicates microorganism growth. Microorganism densities were adjusted to $1.5 \times 10^{8}$ CFU. $\mathrm{mL}^{-1}$. Compound lle, bearing a carboxylic moiety in the benzodiazepine benzene ring was active against all evaluated bacteria (CIM 0,254 to $4,06 \mathrm{mM}$ ), in comparison with its analog le that inhibited only Bacillus cereus (CIM 4,05 mM).

\section{CONCLUSION}

The 4-aryl-4,5-dihydro-1H-1,5-benzodiazepin-2-ol derivatives can be afforded by the reaction between arylidenmalonates of Meldrum's acid and 0 phenylendiamines. Derivative Ile, exhibiting the carboxylic moiety was found to be more active than analog le.

\section{ACKNOWLEDGEMENTS}

Authors greatly acknowledge CAPES/MES 09-61 project) for financial support.

\section{REFERENCES}

1. Nicolaou K. C., Pfefferkorn J. A., Roecker A. J., Cao G.-Q., Barluenga S., Mitchell H. J., J. Am. Chem. Soc., 2000, 122, 9939.

2. Horton D. A., Bourne G. T., Smythe M. L.., Chem. Rev., 2003, 103(3), 893 